\title{
Glottopol
}

Revue de sociolinguistique en ligne

$35 \mid 2021$

La langue à l'école, de l'institution à la classe : quelles conceptions, quelles normes, pour quels usages?

\section{Regards sur le discours scolaire : saisir des conceptions de la langue et de son enseignement}

Thierry Pagnier et Belinda Lavieu-Gwozdz

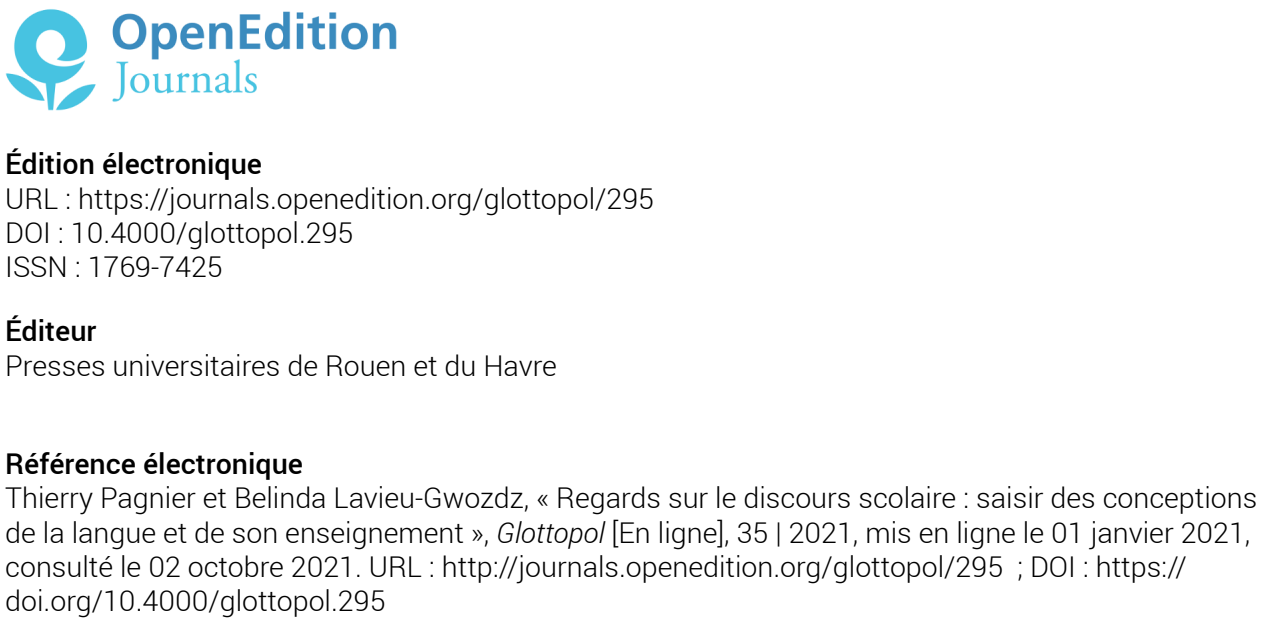

Glottopol 


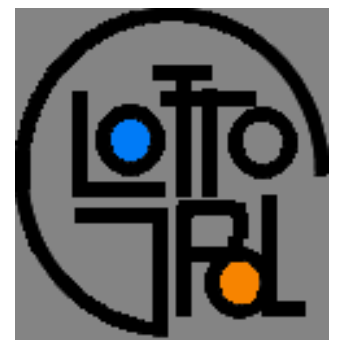

\section{GLOTTOPOL}

Revue de sociolinguistique en ligne $\mathrm{n}^{\circ} 35$ - janvier 2021

\section{La langue à l'école, de l'institution à la classe : quelles conceptions, quelles normes, pour quels usages?}

\section{SOMMAIRE}

Catherine Delarue-Breton et Élisabeth Bautier : Introduction

Catherine Delarue-Breton : Des conceptions scolaires de la langue en tension : l'exemple du programme de 2015 pour l'école primaire et le collège

Véronique Miguel-Addisu : "Sport de riche je l'ai changé en sport élitiste » : plurilinguisme et variation dans la langue de l'école du point de vue des élèves

Claire Colombel-Teuira, Véronique Fillol : Variation et approche polynomique : pour une conception plurielle de la langue à l'école calédonienne

Daphné Bloch : Pratiques langagières, situations pédagogiques et construction d'inégalités d'apprentissage à Madagascar

Samatar Abdallah Doualeh : Le français langue de l'école djiboutienne ou l'hégémonie de la norme exogène

Élise Vinel, Élisabeth Bautier : Des discours des élèves sur l'orthographe aux pratiques des enseignants, analyse d'entretiens métagraphiques

Thierry Pagnier, Belinda Lavieu-Gwozdz: Regards sur le discours scolaire: saisir des conceptions de la langue et de son enseignement

Jacques Crinon, Georges Ferone, Hélène Font : Les enseignants et l'orthographe, une enquête au cycle 3

Christel Troncy : Les enseignants, la norme scolaire et la pluralité langagière dans deux écoles immersives à programme français en Californie. Dynamiques des attitudes et des pratiques lors d'une recherche collaborative

Erwan Le Pipec: L'école, planche de salut du breton?

Argia Olçomendy : La langue basque dans les instructions officielles (1976-2019)

\section{Compte rendu de lecture}

Par Katrin Pfadenhauer: Ursula Reutner (Ed.), 2017, Manuel des francophonies, Berlin/Boston, de Gruyter, 745 p.

Par Robert Fournier: Shana Poplack, 2018, Borrowing: Loanwords in the speech community and in the grammar. New York: Oxford University Press ; xxi, $246 \mathrm{p}$.

Par Marie-Jeanne Verny: Catherine Adam, 2020, Bilinguisme scolaire. Familles, écoles, identités en Bretagne, éd. Peter Lang, coll. «Langue, multilinguisme et changement social », Berlin. 


\title{
REGARDS SUR LE DISCOURS SCOLAIRE ${ }^{1}$ : SAISIR DES CONCEPTIONS DE LA LANGUE ET DE SON ENSEIGNEMENT
}

\author{
Thierry Pagnier \& Belinda Lavieu-Gwozdz \\ Université Paris Est, laboratoire CIRCEFT-Escol \& Université Paris 8, \\ UPEC
}

\section{Introduction}

Le travail présenté ici s'inscrit dans une recherche menée depuis plusieurs années au sein d'un laboratoire de recherche (CIRCEFT-ESCOL) qui centre ses préoccupations sur la question des inégalités d'apprentissage à l'école, et notamment sur le rôle du langage et des discours dans la construction de ces inégalités. Au cœur de ces travaux figure le concept de coconstruction des inégalités scolaires (Bautier 2001 ; Bautier \& Rayou 2009 ; Rochex \& Crinon 2011 ; Delarue-Breton 2012, 2014 et 2015). Les inégalités entre élèves - élèves familialement francophones -, et donc les difficultés importantes, conduisant parfois à l'échec de certains d'entre eux, s'analysent comme étant le produit d'une rencontre entre des modes de socialisation familiale peu connivents des pratiques littéraciées scolaires, et des pratiques pédagogiques qui contribuent parfois à opacifier les enjeux des activités d'apprentissages. Ces processus de co-construction se nourrissent de ce que Bautier, Rayou et Rochex nomment des malentendus sociocognitifs. Le concept de malentendu sociocognitif invite à prendre en compte le travail interprétatif de l'apprenant. Nous prolongeons cette préoccupation en nous intéressant $\mathrm{au}$ sens que les élèves et les enseignants ont effectivement construit des situations d'enseignement-apprentissage lorsqu'ils travaillent l'orthographe du français à l'école élémentaire.

Nous proposons dans cette contribution de mettre en évidence la manière dont les conceptions et les représentations ${ }^{2}$ de la langue française et de son enseignement sont construites et donc observables dans le discours scolaire. Nous avons choisi de nous intéresser à ces représentations et ces conceptions telles qu'elles sont saisissables par l'analyse de ce que les élèves font discursivement à partir des questions qui leur sont adressées. Les élèves identifient-ils des catégories grammaticales, expliquent-ils la procédure utilisée ? Mentionnent-

\footnotetext{
${ }^{1}$ Nous entendons dans cette contribution le « discours scolaire » comme recouvrant à la fois ce qui se réalise dans les programmes 2015, les manuels scolaires de français ou encore des moments saisis dans la salle de classe, etc.

${ }^{2}$ La distinction n'est pas interrogée ici. Sans trancher sur l'intérêt de celle-ci ou questionner la nature de ce qui est à distinguer ici, notre analyse s'intéresse à ce qui peut correspondre tant aux représentations que par exemple Giordan associe à l'ordre du spontané qu'aux conceptions comme ensemble d'idées coordonnées qui prend sens dans « une structure mentale sous-jacente » (Giordan 1987) des élèves comme des enseignants participant à la production de ce discours.
} 
ils les régularités de la langue ? Nous avons cherché à comprendre la manière dont les activités font normes telles qu'elles sont discursivement présentées dans les manuels et telles qu'elles sont langagièrement conduites en classe. Cette double entrée par les manuels et par les interactions a pour finalité de décrire la nature et l'articulation des objets de questionnements dans le discours scolaire pour mieux comprendre la manière dont à travers les habitudes discursives orales et écrites construites dans le cadre de l'école élémentaire se donnent à voir des conceptions du fonctionnement de la langue et de son enseignement.

\section{Méthodologie de la recherche et corpus}

\section{Le questionnement comme observable des conceptions}

Les discours que nous nous proposons d'analyser correspondent à un espace restreint du champ du questionnement tel que le décrit par exemple Maulini (2004). Ces manuels et ces moments de corrections collectives donnent effectivement lieu à des questions qui ne sont pas elles-mêmes sujettes à question et le litige quant aux réponses apocritiques proposées est tranché par une validation. Notre propos ne vise donc pas à interroger les fonctions, la forme et la nature du questionnement mais plus modestement à décrire ce que font discursivement les élèves à partir des questions qui leur sont adressées. Ce n'est donc pas tant le couple questionréponse que l'activité discursive effectivement réalisée que nous cherchons à analyser.

Parmi les pratiques littératiées, le questionnement sur la langue en contexte scolaire est un enjeu constant dans les pratiques pédagogiques et dans la littérature scientifique (Gagnon \& Péret 2009 ; Lavieu-Gwozdz \& Pagnier 2017 ; Pellat 2017). La didactique du français langue maternelle a pointé les limites des opérations d'identification et de repérage en insistant sur l'effet positif d'une verbalisation des procédures utiles à ces identifications (Elalouf 2005 ; Gauvin 2014 ; Nadeau \& Fisher 2011) en promouvant un travail sur les régularités de la langue (Garcia-Debanc et al.2014). Il ne s'agit pas nécessairement d'une rupture dans le type d'activité mais plutôt d'un déplacement de la visibilité et donc l'importance relative des visées de l'activité. Un consensus relativement large dans le champ de la didactique du français invite donc à compléter, voire à inverser la centralité des tâches d'identification et de repérage pour privilégier la verbalisation des procédures de l'analyse linguistique qui les permettent et/ou les nécessitent. Plus que le repérage d'un groupe ou l'identification de la nature ou de la fonction d'une unité, c'est le raisonnement qui a conduit et/ou qui nécessite ce repérage ou cette identification qui est mis en avant; en d'autres termes le raisonnement grammatical (Boutet, 2005).

La mise en mot des procédures est jugée d'autant plus importante que celle-ci permet de rendre visible et possible l'activité intellectuelle et langagière visée. Pour ne pas être réduits à de simples étiquettes, les métatermes de l'analyse grammaticale doivent s'inscrire dans une activité métalinguistique qu'ils rendent possible. Les recherches sur les inégalités sociales et scolaires en contexte français métropolitain ont ainsi montré comment, au-delà des mots, ce sont les usages langagiers qui permettent d'apprendre qui sont inégalement partagés par les élèves (Bautier 1997). Concernant le fonctionnement de la langue ${ }^{3}$, il est alors question pour

\footnotetext{
${ }^{3} \mathrm{Au}$ sens le plus courant, une langue est un instrument de communication, un système de signes vocaux spécifiques aux membres d'une même communauté. Au sein d'une communauté linguistique donnée tous les membres de cette communauté (tous les locuteurs du français, par exemple) produisent des énoncés qui, en dépit des variations individuelles, leur permettent de communiquer et de se comprendre, et qui reposent sur un même système de règles et de relations qu'il est possible de décrire. C'est à ce système abstrait, sous-jacent à tout acte de parole que F. De Saussure a donné le nom de langue. Nous définissons ici le terme de langue, volontairement au singulier comme un instrument de communication et de pensée qui fonctionne à la manière d'un système de signes et nous nous
} 
les élèves de faire de la langue non seulement un objet de discours (Brissaud \& Mortamet 2015 ; Hubert 2016) mais plus spécifiquement l'objet d'un discours d'analyse, nécessitant une évolution plus ou moins profonde de leur rapport à la langue et à son fonctionnement. L'opacité de ce réquisit pour certains élèves peut ainsi être pointée au titre des malentendus sociocognitifs qui expliquent les processus de co-construction des inégalités sociales et scolaires. Enfin, audelà d'une recommandation, bien ancienne, à travailler du « courant au rare » en raison d'une logique qui se voudrait naturelle, de nombreuses recherches insistent sur le lien entre l'absence de perception des régularités de la langue et les difficultés rencontrées en étude de la langue. L'idée n'est pas nouvelle: les programmes officiels appelaient, dans les années 2000, « Observation Réfléchie de la Langue », les séances dites ordinairement de grammaire.

Pour rendre compte des conceptions et représentations de l'enseignement-apprentissage du fonctionnement de la langue française qui se construisent en classe élémentaire (FLM), nous nous proposons donc d'analyser à quelles activités discursives donnent lieu les questions et les réponses qui y sont apportées en distinguant l'étiquetage, la verbalisation des procédures et la verbalisation des régularités de fonctionnement de la langue.

\section{Le questionnement dans les programmes}

La mise en place à la rentrée 2016 de nouveaux programmes pour les cycles 2 , 3 et 4 nous a semblé un point de départ intéressant à explorer lorsqu'on cherche à rendre compte de la manière dont l'institution et les enseignants se représentent la langue. Ces programmes semblent marquer une rupture concernant l'enseignement de celle-ci. En effet, au-delà de 1'aspect formel lié à la réorganisation des cycles (CE2 ou $3{ }^{\text {ème }}$ primaire devenant la fin du cycle 2 entraine de fait un changement au niveau des attentes sur la langue), la lecture de ces textes institutionnels révèle plusieurs évolutions :

- un enseignement de la langue qui doit se coordonner et s'organiser du CP (cours préparatoire, $1^{\text {ère }}$ primaire) à la classe de troisième ;

- la langue doit s'enseigner à partir de ses régularités et on accorde une importance à l'observation et la manipulation des énoncés (reprenant ainsi l'ORL des programmes de 2002);

- le lien avec l'oral ainsi qu'avec les activités du lire-écrire pour l'ensemble des trois cycles est fortement affirmé ;

- approcher la langue et son fonctionnement dans son ensemble et non de façon cloisonnée ;

- la terminologie dans le domaine de l'étude de la langue doit être réduite et explicite s'appuyant davantage sur les caractéristiques syntaxiques des unités plutôt que sur leur sens.

D’une manière générale, ces programmes invitent à un changement de posture tant de la part des élèves que de celle des enseignants (Audion 2015). En effet, de nombreuses études (Chartrand 2016; Gauvin 2014) indiquent que pour beaucoup d'élèves l'orthographe est difficile, il faut apprendre des règles, des exceptions par cœur et de nombreux enseignants confient à leur tour que c'est un domaine difficile à enseigner, dans lequel ils ne sont pas sûrs d'eux (y compris des professeurs de lettres du secondaire) (Gauvin 2014 ; Ulma 2016). L'enjeu des programmes de 2015 s'agissant de l'étude de la langue est « véritablement d'impulser une pratique de l'étude de la langue, et non d'accumuler des savoirs grammaticaux » (Duez \& Risselin 2017: 26). Cet élément est un marqueur fort de l'orientation d'un texte programmatique qui cherche moins la rupture que le déplacement. " Il n'y a ni grammaire nouvelle, ni révolution [...] mais un déplacement du centre de gravité de l'enseignement de la langue » (ibid.). En effet, les nouveaux programmes impliquent de rompre avec le cumul de

préoccuperons ici exclusivement du français langue maternelle en contexte métropolitain dans l'espace de la classe. 
connaissances grammaticales comme visée de l'enseignement de la grammaire. Les programmes de 2015 proposent, eux, en matière d'étude de la langue une perspective d'apprentissage, et non plus d'enseignement. Le centre d'attention se déplace des notions de la grammaire scolaire sur lesquelles le professeur devait fonder son enseignement, aux capacités que l'on va chercher à développer chez les élèves. Ce qui sous-tend les programmes dans leur globalité pour l'étude de la langue est donc « la nécessité d'encourager les élèves à adopter une posture métaréflexive pour comprendre les régularités au cœur du fonctionnement de la langue » (Audion 2017), comprendre que la langue fait système. Le terme est martelé dans les programmes de 2015 ainsi, dans la partie « Étude de la langue », on peut lire qu'un des objectifs importants est « de mettre en évidence les régularités et de commencer à construire le système de la langue ». Du point de vue didactique, cela n'est pas nouveau. Ce qui l'est en revanche, c'est l'institutionnalisation de cette vision.

Selon Duez \& Risselin (2017), la partie « compétences linguistiques » des programmes de 2015 de cycle 4 rappelle que :

l'enseignement explicite des gestes du grammairien (supprimer, déplacer, remplacer, ajouter, cliver...) passe par des activités linguistiques de haut niveau. Les propositions extraites des nouveaux programmes (observations/comparaisons, manipulations syntaxiques, emploi et réemploi...) invitent les élèves à raisonner sur la langue : le travail sur corpus de phrases, les situations-problèmes à résoudre en groupe, les bilans de savoir accroissant la réflexivité sont autant de pistes de travail collaboratif, dynamiques, susceptibles de déplacer certaines représentations tout au long du cycle.

En somme, les programmes de 2015 s'écartent manifestement des programmes de 2008 en affichant une volonté d'enseigner explicitement l'étude de la langue par le recours à une méthode scientifique sur le modèle de ce qu'auraient proposé les documents d'accompagnements associés aux programmes 2002 en «Observation Réfléchie de la Langue ». Néanmoins, ces programmes ne proposent pas une démarche spécifique de questionnement sur la langue.

\section{Le questionnement dans deux corpus : les manuels et les interactions en groupe classe}

Si les programmes invitent effectivement au questionnement grammatical en classe, le questionnement lui-même ne fait pas l'objet d'une normalisation explicite. Nous avons donc voulu apprécier la normativité de l'action de questionnement, c'est-à-dire la norme en acte ${ }^{4}$.

Un premier corpus est constitué d'extraits de manuels qui ont en commun de mettre en avant le «questionnement» dès leur sommaire. Nous avons ainsi sélectionné trois des derniers manuels scolaires de français récents utilisés par nos étudiants et stagiaires à l'INSPÉ de Créteil qui présentaient cette caractéristique : Terre des mots, CM1 (cycle 3), Explorons la langue, CM2 (cycle 3) et Interlignes, CM2 (cycle 3). Ces trois manuels ont également comme caractéristiques de travailler uniquement l'étude de la langue et non la littérature. Au-delà des lignes éditoriales et des collectifs qui ont contribué à chacun d'eux et qui éclairent les choix didactiques, nous avons choisi de questionner leur réception par les étudiants stagiaires. Nous avons donc d'abord fait primer la manière dont les étudiants stagiaires se saisissaient de ces manuels notamment par prélèvement de doubles pages, voire d'exercices. Pour chacun d'eux, nous avons donc sélectionné deux chapitres en lien avec l'orthographe grammaticale qui permet

\footnotetext{
${ }^{4}$ À la suite de N. Ramognino, nous nous intéressons ici à la normativité de l'action en reprenant le cadre de son hypothèse de « la norme comme outil cognitif et celle de l'institution comme dispositif cognitif collectif » (2007: 39). La routinisation des pratiques en l'occurrence discursives constitue donc, selon nous, un observatoire propice à l'analyse des représentations et des conceptions.
} 
de voir quelles activités sont proposées aux élèves (restitution de savoir, mobilisation du métalangage, procédures, manipulations linguistiques, etc.) et donc quels objets de questionnement elles visent. Il s'agit respectivement pour le premier manuel (Terre des mots) des chapitres "Comment ${ }^{5}$ repérer le sujet du verbe " que l'on trouve classé dans le domaine [grammaire] (chapitre 4, p. 25) et «Comment accorder le verbe avec le groupe nominal sujet » classé dans le domaine [orthographe] (chapitre 5, p. 130), pour le deuxième manuel (Explorons la langue) des chapitres «Pourquoi et comment repérer le sujet du verbe conjugué » (chapitre 6, p. 33) et "Comment choisir la bonne marque de personne » (chapitre 19, p. 89) et pour le manuel Interlignes, les chapitres «Le sujet (1) : comment reconnaitre le sujet? 》, classé dans le domaine [grammaire] (chapitre 3, p. 18) et "L'accord $d u$ verbe avec le sujet: comment accorder le verbe avec le sujet ? ", classé dans le domaine [orthographe] (chapitre 9, p. 120). Nous nous sommes intéressés aux consignes en identifiant l'objet du questionnement qu'elles visaient en distinguant les demandes d'étiquetage par identification de la nature ou de la fonction d'un mot ou d'un syntagme (Quelle est la fonction de X? / c'est un X) et par repérage de la nature ou de la fonction d'un mot ou d'un syntagme dans une phrase (Quel est le sujet de cette phrase?/X est le sujet de la phrase), des demandes explicites d'application ou de verbalisation de procédures et les demandes de déduction ou de mémorisation des régularités de la langue.

Le second corpus est constitué de la transcription de séances de correction de dictées et d'exercices d'orthographe de classe de cycle 3 (CM1-CM2) dont nous proposons d'analyser le questionnement avec les mêmes catégories. Il s'agit d'observations « écologiques », c'est-àdire avec l'intervention la moins sensible possible sur les manières habituelles de faire et de dire, en intervenant le moins possible dans le dispositif et l'environnement. Quelle que soit la variété des ingénieries didactiques qu'avaient choisies les enseignants (Twictée, dictée inversée, leçon et exercices de grammaire), toutes prévoyaient une correction collective des dictées et exercices individuellement produits sous forme de cours dialogué. Le recueil de données a été effectué de 2017 à 2019 par l'ensemble des membres du projet E-Fran TAO auprès de 40 classes $^{6}$. Les séances de classes ont été filmées à l'aide d'une caméra positionnée sur un plan large de sorte que les interactions soient visibles et audibles et transcrites (normes du GARS aménagées). Dans le cadre de cette contribution, nous présentons les résultats de nos analyses exploratoires sur un corpus de cinq classes au cours d'une séquence de cinq séances sur l'orthographe. Parmi ces cinq classes, trois relèvent d'un Réseau d'Éducation Prioritaire (REP) tandis que les deux autres se trouvent hors-REP. La perspective comparative sera développée ultérieurement lorsqu'un nombre plus important de séances de classe aura été analysé.

\section{La conception de la langue dans les manuels de 2018}

Il s'agit pour nous d'explorer les manuels pour ce qu'ils révèlent de leur discipline, des enjeux de leur élaboration, de leur transformation. Selon Chervel (1988 : 56), les manuels sont " des contributeurs importants dans l'élaboration de la culture scolaire ». L'étude des manuels apporte des informations éclairantes sur les évolutions du français comme discipline et ses contenus. Cette approche nécessite cependant une certaine prudence car le manuel ne peut être

\footnotetext{
${ }^{5}$ Nous mettons volontairement ces mots interrogatifs en évidence.

${ }^{6}$ Projet Espaces de formation, de recherche et d'animation numérique (e FRAN), financement Programme d'Investissements d'Avenir (PIA), porté par l'université Paris Est Créteil. Voir : https://inspe.u-pec.fr/rechercheet-innovation/recherche-et-internationalisation/contrats-et-projets-de-recherche/tao-renforcer-les-competencesen-orthographe-avec-le-dispositif-twictee .
} 
considéré comme un simple reflet des pratiques dont il permettrait de saisir la réalité ou d'une transposition transparente et neutre des préconisations :

Les manuels ne sauraient apparaitre non plus comme des reflets des prescriptions dont ils ne sont que des interprétations [...]. Les manuels sont "polyphoniques " c'est-à-dire à la fois porteurs de savoirs, de documentations foisonnantes, d'outils méthodologiques, il leur faut en outre tenir compte de publics hétérogènes. (Denizot \& Bishop $2016: 7$ )

La conformité avec les programmes en vigueur est néanmoins un argument éditorial fort qui est affiché dès la couverture de l'ensemble des manuels examinés. Deux des trois manuels que nous avons retenus font par ailleurs explicitement référence aux programmes dans leur avantpropos. Alors que le manuel Explorons la langue met en avant les régularités de la langue avec au centre de ses préoccupations les compétences en orthographe : «Les chapitres du manuel s'appuient sur des phénomènes réguliers en orthographe, en conjugaison, en grammaire et sur le lexique sélectionné [...] un accent est porté sur le but orthographique de toute activité d'étude de la langue », le manuel Terre des mots met, lui, en avant une progression devant aller de « la simplicité à la complexité » et du " fréquent au rare » sans que cette proposition ne soit par ailleurs étayée : «Proposer une étude réfléchie de la langue en allant du plus simple au plus complexe et du plus fréquent au plus rare ». La conformité avec les programmes ouvre donc des propositions pédagogiques différentes selon l'importance relative accordée aux déplacements identifiés dans les programmes et sélectionnés par les auteurs et les éditeurs.

\section{Discours classificateur : domaine et sous-domaines}

Les choix effectués par les auteurs/éditeurs dans les différentes configurations disciplinaires, notamment en ce qui concerne le découpage en domaines ou sous-domaines que l'on trouve dans le sommaire, parfois regroupés, parfois séparés, ne sont pas sans incidence sur la conception même de la langue et de la discipline qu'est le français. Il s'agit de s'intéresser à ce que Bernstein appelle le discours classificateur (Bernstein 2007).

Le sommaire de Terre des mots propose une distinction en quatre domaines : grammaire, conjugaison, orthographe et vocabulaire, bien étanches sans interaction d'un domaine à l'autre. Ainsi le chapitre "Comment repérer le sujet $d u$ verbe » à la page 25 se trouve classé dans la partie grammaire tandis que le chapitre «Comment accorder le verbe avec le groupe nominal sujet » se trouve à la page 130 dans le domaine orthographe. À aucun moment le manuel ne propose de renvois de chapitres permettant de rendre visibles les articulations entre grammaire et orthographe. On retrouve dans le sommaire d'Interlignes le même type d'organisation en quatre sous-domaines complétés par un chapitre sur le vocabulaire des disciplines scolaires.

À l'inverse, le sommaire de Explorons la langue propose un autre classement en plaçant l'orthographe au centre des compétences de toute activité d'étude de la langue. Ainsi l'orthographe est systématiquement couplée tantôt avec la grammaire, tantôt avec la conjugaison tantôt avec le lexique. Par exemple le chapitre "Pourquoi et comment repérer le sujet du verbe conjugué » est rangé dans le domaine grammaire-orthographe tandis que celui intitulé "Comment choisir la bonne marque de personne » est classé dans le double domaine conjugaison-orthographe. Comme dans Terre des mots, les deux chapitres sont distincts l'un de l'autre de plusieurs pages, l'un se trouve, respectivement p. 33 l'autre, p. 89 sans qu'un renvoi signale aux élèves que ces deux chapitres peuvent être articulés.

Ce qui a également retenu notre attention dans ces trois manuels récents est la formulation même du chapitre par des interrogatifs comment? pourquoi? qui interrogent donc, linguistiquement parlant, sur le système de la langue, sur la manière dont la langue fonctionne. Les interrogatifs présents dès le sommaire peuvent se lire comme une traduction de la rupture entre l'accumulation de savoirs grammaticaux qui étaient privilégiée dans les anciens 
programmes et la posture méta-réflexive qui est maintenant demandée aux élèves censés conduire une "observation » de corpus ; c'est-à-dire manipuler les éléments linguistiques. L'entrée par la procédure fait aussi certainement écho à la place grandissante de la métacognition dans le champ des didactiques notamment en français dans l'apprentissage de la lecture (Cèbe \& Goigoux 2009) et de l'orthographe (Cogis 2003 ; Nadeau \& Fisher, 2011).

\section{Les consignes : des objets de questionnement inégalement représentés}

Les énoncés d'exercices et leurs consignes ne sont pas transparents. Ils relèvent d'un code particulier qui peut faire écran à la tâche demandée, et nécessitent donc l'apprentissage d'une compétence textuelle préalable à une lecture efficace. Les énoncés d'exercices sont injonctifs c'est-à-dire qu'ils visent à faire accomplir une certaine tâche mais l'enjeu didactique de la consigne est le plus souvent masqué. Pour avoir conscience de cet implicite, il faut avoir une certaine représentation de l' "interlocuteur » (l'enseignant à qui on adresse l'exercice, par exemple), de la place et du rôle respectif de l'institution, de l'enseignant et de l'enseigné : ce dernier, malgré les apparences, ne doit pas être seulement capable de trouver une réponse : il doit, de surcroit, être en mesure de tenir un raisonnement, montrer comment il a trouvé cette réponse en somme, prouver qu'il a compris sans que cela n'apparaisse nécessairement de manière explicite. Nous postulons que la normativité de l'action qui s'exerce en discours par la répétition des formats et des objets de questionnements dans ces situations d'enseignementapprentissage participe de la construction des représentations du fonctionnement de la langue et plus largement du rapport au langage que construisent les élèves.

Pour opérer cette analyse, nous avons identifié les trois pôles de questionnement: l'identification et le repérage, les procédures à appliquer et l'observation des régularités de la langue en analysant les consignes. L'histogramme ci-dessous indique les données quantitatives obtenues après traitement de 123 consignes visant 154 objets de questionnement pour l'ensemble des trois manuels étudiés. Une consigne du type «Encadre les sujets par "c'est ... qui" ou "ce sont ... qui" " est donc catégorisée comme proposant un questionnement à la fois sur le repérage et sur l'application de procédures. Une analyse complémentaire pourrait par ailleurs interroger l'articulation plus ou moins explicite entre l'identification et la procédure (et entre la procédure et le fonctionnement de la langue). En l'occurrence, s'agit-il de repérer les sujets puis de les encadrer ou de procéder au test d'encadrement pour repérer les sujets? Néanmoins, il s'agit d'un des rares exemples où le repérage est " associé » à une procédure.

Nous avons ajouté une catégorie relative aux tâches de manipulations et de transformations qui n'impliquent pas nécessairement et explicitement une procédure d'analyse comme dans l'exemple suivant extrait du manuel Explorons la langue: "Remplace le nom ou le groupe nominal sujet par un pronom personnel. »

\section{Au centre du questionnement : l'étiquetage (repérage et identification)}

Ce premier ensemble vise l'identification tantôt de la classe grammaticale de mots, de la fonction, de la bonne forme orthographique, c'est-à-dire du «quoi ». Cette catégorie est massivement représentée dans notre corpus sur les manuels scolaires de cycle 3 quel que soit le manuel puisqu'elle occupe au minimum la moitié des objets de questionnement (Explorons la langue) et jusqu'à deux tiers des objets de questionnement dans Terre des mots. 


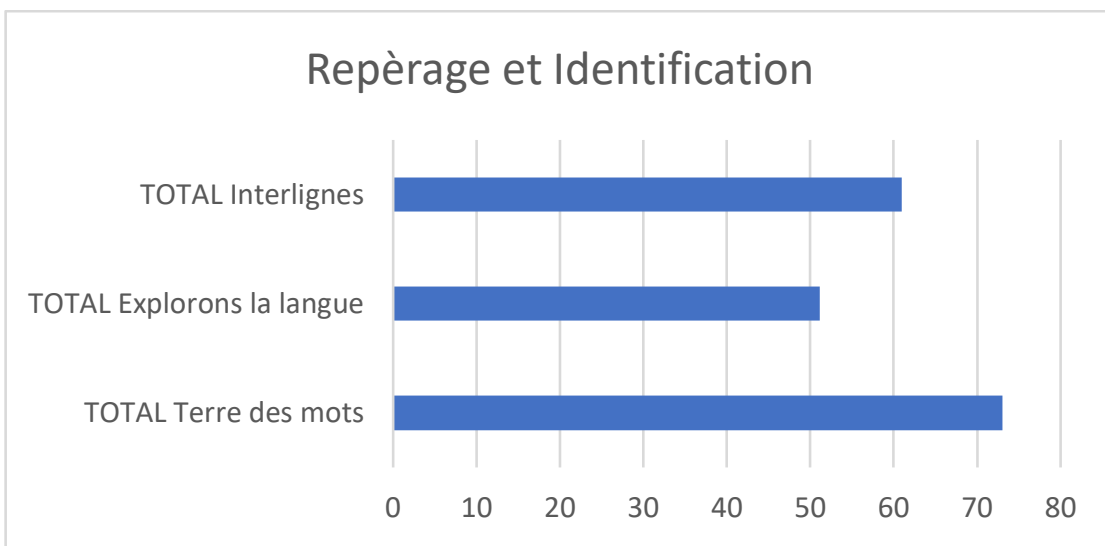

Figure 1 : Part des consignes portant sur le repérage et l'identification dans les trois manuels

Nous regroupons dans ce premier ensemble les formulations des consignes d'exercice suivantes: Indique la classe grammaticale, Pourquoi a-t-on mis des mots en gras dans ce texte ?, Complète les phrases avec les mots de la liste en respectant la chaine d'accord. Ici, il s'agit, pour les élèves, d'identifier tantôt la classe grammaticale, tantôt de repérer la fonction des mots par des jeux de couleurs (sujet en bleu, le verbe en rouge, etc.), tantôt de compléter des phrases avec les mots d'une liste imposée. On voit donc que pour ce premier ensemble (indiquer, repérer, souligner, quel/quelle, etc.), il s'agit bien pour les élèves de se prononcer sur le /QUOI/ c'est-à-dire que l'on vise l'identification sans que la question ne soit d'ailleurs nécessairement formulée par l'introducteur linguistique «quoi ». Ainsi, la formulation de la consigne vise comme réponse une étiquette soit du morphème, soit du graphème soit de la nature grammaticale ou encore de la fonction. La consigne peut également viser la réponse orthographique ou grammaticale c'est-à-dire la bonne forme sans faire justifier ou argumenter les réponses. L'activité de raisonnement orthographique et/ou grammatical est ainsi potentiellement opacifiée par l'exécution de la tâche de repérage ou d'identification. Au-delà de la focalisation de certaines consignes sur la part matérielle de l'activité plutôt que sur l'activité intellectuelle (souligner/entoure $v s$ repérer/identifier...) attendue, ce sont effectivement les visées de l'activité qui risquent de ne pas être visibles pour tous dès lors que dans le discours scolaire les tâches d'identification et de repérage ne prennent pas sens dans les questionnements qui les nécessitent et/ou les rendent possibles.

\section{Les procédures à appliquer ou à expliquer : une part variable du questionnement}

Nous repérons un second ensemble dans les consignes données au sein des activités proposées dans ces manuels. Ce second ensemble, nous l'avons appelé /COMMENT/ dans la mesure où il ne s'agit plus ici d'identifier mais davantage de manipuler et de mentionner des procédures. Là encore, comme pour notre premier ensemble, la forme linguistique ne présage pas de ce qui est attendu et la forme en comment n'interroge pas toujours sur le comment. Pour l'illustrer, la formulation en comment dans le manuel Terre des mots pour une consigne telle que Comment sais-tu à quel élément du dessin se rapporte chaque paragraphe du texte? (p. 25) est en fait de l'identification, du /QUOI/ (notre premier ensemble), alors que la consigne de l'exercice 6 p. 34 Explique comment tu as fait pour trouver le sujet dans Explorons la langue, vise bien ici notre ensemble en /COMMENT/dans la mesure où l'on attend de l'élève ici qu'il mobilise des procédures linguistiques, par exemple l'encadrement en c'est ... qui ou ce sont ... $q u i$, mentionné dans le texte de savoir, explicitant ainsi la manière dont il s'y est pris pour repérer le sujet. En somme, bien que les deux manuels intitulent leurs chapitres avec les interrogatifs Comment ou Comment et pourquoi..., les deux ne semblent pas procéder de la même manière et mobiliser le même savoir et savoir-faire sur la langue chez les élèves. En effet, le tableau ci-dessous indique que les consignes qui visent le /COMMENT/ représentent 
respectivement $15,5 \%$ et $17 \%$ des objets de questionnement dans Explorons la langue et Interlignes alors qu'elles ne sont que $7,9 \%$ dans Terre des mots.

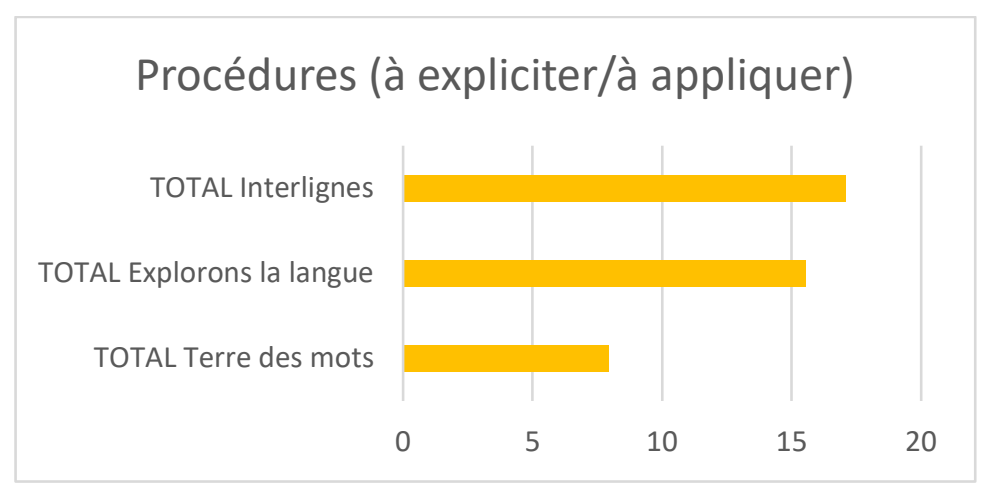

Figure 2 : Part des consignes portant sur les procédures dans les trois manuels

Ainsi, Terre des mots semble davantage demander aux élèves de mobiliser des connaissances sur la langue plutôt que de faire fonctionner cette langue par le biais de manipulations.

Les régularités de la langue et les manipulations/transformations syntaxiques

Comme pour nos deux précédents ensembles, nous ne nous sommes pas arrêtés à l'analyse de la forme des questions pour repérer cet ensemble dans la mesure où une question formulée en pourquoi ne visait pas toujours l'argumentation. Ainsi, à titre d'illustration, l'exercice 3 p. 33 dans Explorons la langue formule la consigne suivante Transforme les phrases en couleur. Réécris la phrase en orange en remplaçant cette petite fille par ces petites filles. Réécris la phrase en violet en remplaçant des voyageurs par un voyageur. As-tu modifié les verbes? Pourquoi? invite effectivement les élèves à justifier/argumenter leurs choix orthographiques et le manuel le fait de manière explicite en évoquant la relation avec le verbe (As-tu modifié les verbes ?). À l'inverse, une même question en pourquoi dans l'exercice 2 p. 25 dans le manuel Terre des mots, Pourquoi a-t-on mis des mots en gras dans le texte?, n'invite pas à justifier ni à argumenter et qui plus est, la formulation ne vise pas directement la notion de sujet. On peut très bien imaginer que certains élèves peu connivents ne tissent pas de lien entre cette consigne et l'intitulé du chapitre et mobilisent davantage de connaissances sémantiques pour répondre à cette question. En effet, les mots en gras (racines, tige, feuilles, fleur et fruits) appartiennent tous à un même champ sémantique si bien que les élèves peuvent totalement passer à côté de l'enjeu d'apprentissage qui est de repérer le sujet du verbe. Quel que soit le manuel, le questionnement portant sur l'identification des régularités de la langue est marginal et n'occupe pas plus de 6 à $8 \%$ des objets de questionnement dans les manuels Interlignes et Explorons la langue tandis que cette catégorie représente moins de $2 \%$ dans Terre des mots.

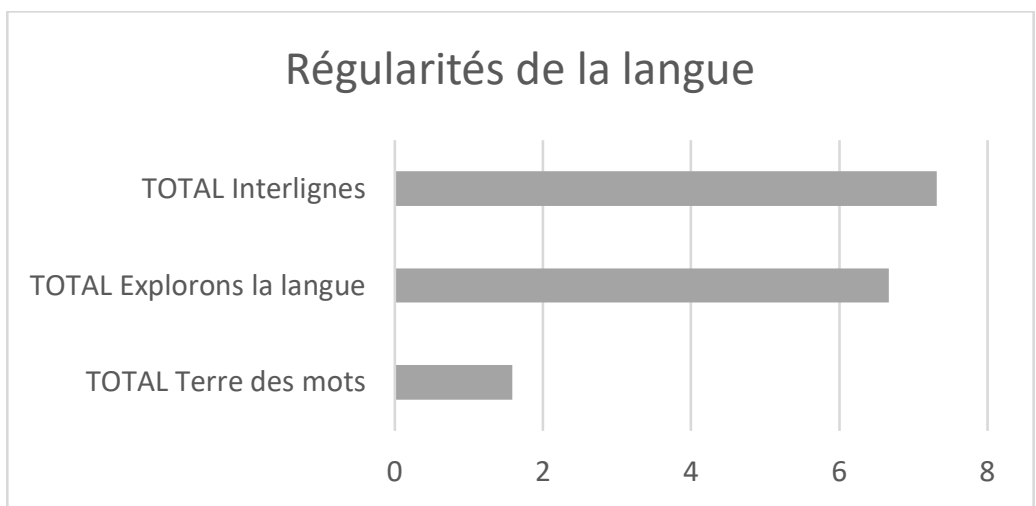

Figure 3 : Part des consignes portant sur les régularités de la langue dans les trois manuels 
Ce qui distingue les trois manuels correspond davantage à des questionnements visant la manipulation ou la transformation d'énoncés. Cela représente environ un quart des consignes dans le manuel Explorons la langue tandis qu'il représente à peine $12 \%$ dans le manuel Interlignes et seulement 6,5\% dans Terre des mots.

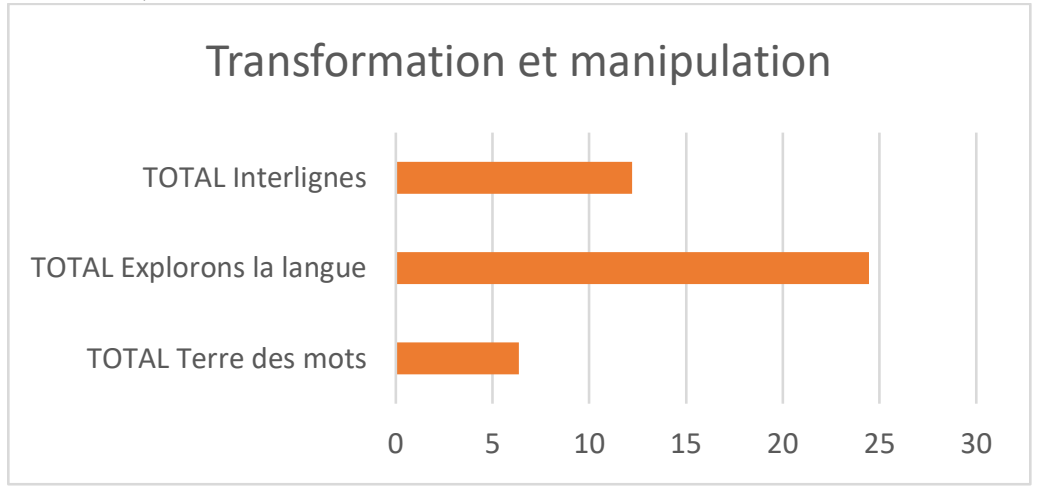

Figure 4 : Part des consignes portant sur les transformations et les manipulations dans les trois manuels

En somme, il semble que le manuel Explorons la langue vise davantage à faire percevoir aux élèves, dans la formulation même des consignes, que la langue fonctionne comme un système, que l'on doit manipuler, expérimenter pour en comprendre les régularités. Terre des mots se caractérise par une prédominance du questionnement visant l'identification ou le repérage qui occupe les trois quarts du manuel. Interligne propose des proportions intermédiaires avec toujours une place majoritairement faite à l'identification et au repérage.

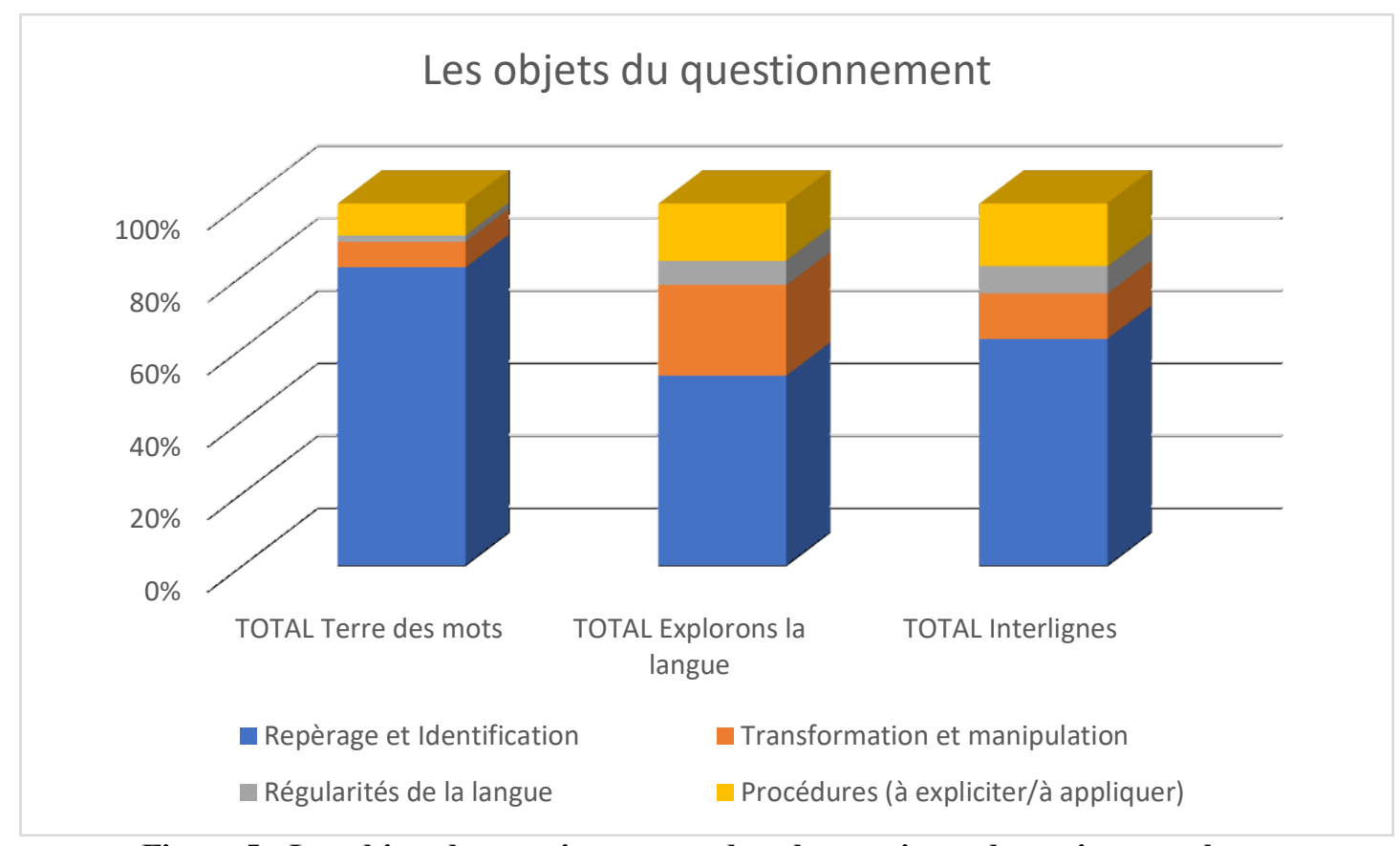

Figure 5 : Les objets du questionnement dans les consignes des trois manuels

\section{Le texte de savoir}

Chaque manuel propose aux élèves (et aux enseignants) un texte de savoir qui répond à la question posée dans le titre du chapitre. Néanmoins, plusieurs éléments les distinguent. D'abord, la place de cet encadré est différente dans les trois manuels : alors que pour Terre des mots et Interlignes, le texte de savoir se trouve à la fin du chapitre, l'encadré de la leçon se trouve juste après la phase de découverte donc au milieu du chapitre pour Explorons la langue 
et est complété par une institutionnalisation spécifique de la procédure à suivre pour accorder le verbe avec son sujet.

Par ailleurs, si tous font état des caractéristiques sémantiques et morphologiques des catégories étudiées, Terre des mots ne propose pas de procédures syntaxiques et par exemple ne mentionne pas l'encadrement par «c'est...qui » pour repérer le sujet. La seule procédure de repérage fournie est d'ordre sémantique (Qu'est-ce que/qui fait l'action ?) pour repérer le sujet.

Cette analyse des manuels mérite d'être mise en perspective avec l'analyse des interactions en classe lors de la correction d'exercices préalablement réalisés individuellement.

\section{La conception de la langue pendant les moments de classe}

Cette troisième partie vise à identifier la représentation de la langue et les conceptions de son enseignement-apprentissage à travers les interactions verbales dans la classe lors d'apprentissages orthographiques (dictées, twictées, exercices d'orthographe). Notre attention s'est tournée vers l'analyse des interactions en groupe classe. À une époque où l'horizontalisation des échanges pose question (Bautier 2005, 2009), nous avons choisi de nous intéresser au discours adressé et construit en groupe classe. Ce choix est motivé par l'idée que les interactions entre pairs, sur lesquelles investissent de nombreux dispositifs pédagogiques contemporains, sont nécessairement articulées à ces moments de mises en commun des réponses et des raisonnements (Douaire et al. 2005). Ces interactions en groupe classe nous semblent se dérouler sur la base de ce que l'on pourrait désigner comme une double illusion. D'une part, l'interaction entre quelques élèves et l'enseignant est un discours également adressé aux autres élèves de la classe. Il s'agit dès lors de se demander dans quelle mesure celui-ci est effectivement saisissable par (tous) les élèves. D'autre part, l'enseignant questionne non (simplement) pour obtenir des réponses mais pour montrer/donner à voir le questionnement que les élèves doivent s'approprier. Il s'agit dès lors de se demander comment cette finalité " seconde », souvent masquée par la place centrale qu'occupe l'exécution de la tâche dans les interactions, est donnée à voir.

Notre intérêt pour décrire les spécificités du dialogue scolaire a été suscité par le désir de mieux comprendre ce qui fait difficulté notamment pour certains élèves dont le mode de socialisation familiale est moins en adéquation avec la socialisation scolaire que d'autres élèves. Un certain nombre de travaux ont montré en effet que les difficultés récurrentes de certains élèves sont directement liées à ce qui se joue dans les échanges langagiers scolaires (voir notamment Bautier 2008 ; Bautier \& Rochex 2004 ; Delarue-Breton 2017). Dans l'histoire de l'école, « la question, c'est d'abord une condition pour que les élèves apprennent, pour qu'ils trouvent du sens et s'engagent dans le travail scolaire » (Maulini 2004 : 127). Nous avons cherché à savoir à quelle activité discursive le questionnement donnait effectivement lieu.

En somme, nous nous intéressons à la manière dont les moments scolaires c'est-à-dire lorsqu'on fait des exercices ou des leçons d'orthographe contribuent ou participent à construire ces représentations de ce que signifie « faire de l'orthographe » ou « faire de la grammaire » et de manière plus générale, des représentations de ce qu'est la langue et de son fonctionnement (Lavieu-Gwozdz \& Pagnier 2017).

\section{Des moments discursifs qui visent l'identification : le /QUOI/}

Dans notre corpus, nous avons donc isolé des moments discursifs qui portent sur l'étiquetage autrement dit le plus souvent sur l'identification des catégories grammaticales. Ce sont ces moments de discours que nous avons enregistrés dans notre catégorie /QUOI/. Nous ne parlons volontairement pas de « question en quoi » parce que nous voyons dans les extraits ci-dessous que pour cette catégorie «identification », nous ne nous sommes justement pas arrêtés 
uniquement aux introducteurs linguistiques. Les questions sont souvent formulées de la manière suivante par l'enseignant : "Qu'est-ce que je mets ? », "C'est quoi ? », « C'est X [morphème de pluriel pour les noms, par exemple] » ou «Y [morphème de pluriel pour les verbes] ». "Ça s'écrit comment? », "C'est un [+ le nom de la catégorie grammaticale] ». La formulation de la question vise une étiquette soit du morphème, soit du graphème, soit de la nature grammaticale.

\section{Extrait $n^{\circ} 1$}

Ens : oui alors c'est parti ++ c'est quoi finit?

E : un verbe

Dans la même catégorie, bien que l'activité cognitive soit différente, nous avons classé les réponses qui visent à faire produire la forme orthographique correcte sans en viser l'explicitation. Ainsi dans l'extrait $\mathrm{n}^{\circ} 1$ bis, Juliette épèle les mots les uns à la suite des autres (quand, volcan) :

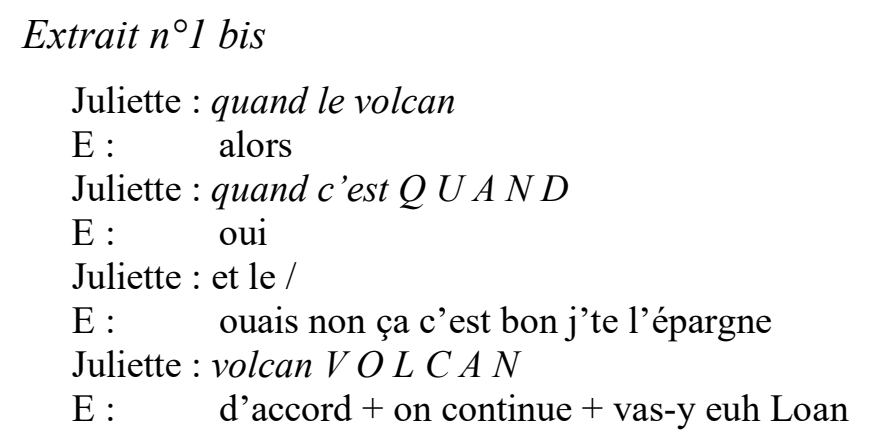

Bien que nous ne nous intéressions pas spécifiquement ici aux gestes et épisodes évaluatifs (Jorro et Mercier-Brunel 2011), nous pouvons noter que cette validation intervient explicitement comme dans l'extrait $\mathrm{n}^{\circ} 1$ ou implicitement par le traitement de la question suivante dans $1^{\prime}$ extrait $n^{\circ} 2$ :

\section{Extrait $n^{\circ} 2$}

Laura : c'est vous riez

M : $\quad$ ah vous riez vous riez Lila Lilia

Lila : c'est aller au passé simple

M : $\quad$ alors aller au passé simple

Édouard : ils allèrent A deux L E accent grave R E NT

$\mathrm{M}: \quad$ il va comment Dimitri euh e présent avec je Jorel

Jorel : $\quad$ c'est le verbe donner je donne D O deux $N E$

Il s'agit ici de corriger des formes orthographiques mais on remarque que la manière de faire est la même pour corriger des items nominaux comme des items verbaux. Ces deux extraits (il s'agit de deux classes différentes) montrent que les enseignants recourent à l'épellation de la (bonne) réponse sans faire justifier ou argumenter ou découper en morphème les réponses des élèves. Ce qui prévaut dans cet échange c'est la (bonne) forme, ce qu'il fallait mettre, quelle était la bonne réponse. L'activité de raisonnement orthographique tend ainsi à être opacifiée par l'exécution de la tâche.

Le découpage du corpus selon ces catégories montre une majorité de questions visant l'identification et cela qu'il s'agisse de classe REP ou non-REP, twictante ou non twictante, du moins dans l'état actuel du traitement et de l'analyse données. 
À titre d'exemple, sur une séance de 45 minutes d'orthographe, 35 minutes concernent la correction de la dictée. En 35 minutes, 13 items (en moyenne) sont passés en revue. Ce temps d'interaction compte 23 questionnements répartis très inégalement (cf. schéma 1), c'est-à-dire des questionnements sur le QUOI - autrement dit comme nous l'avons vu plus haut sur l'étiquetage (identification et repérage auxquels nous avons ajouté la forme orthographique correcte épelée) - et de manière très minoritaire sur le POURQUOI c'est-à-dire sur le fonctionnement de la langue vu comme un système et le COMMENT qui correspond aux moments durant lesquels les élèves énoncent, verbalisent, explicitent des procédures.

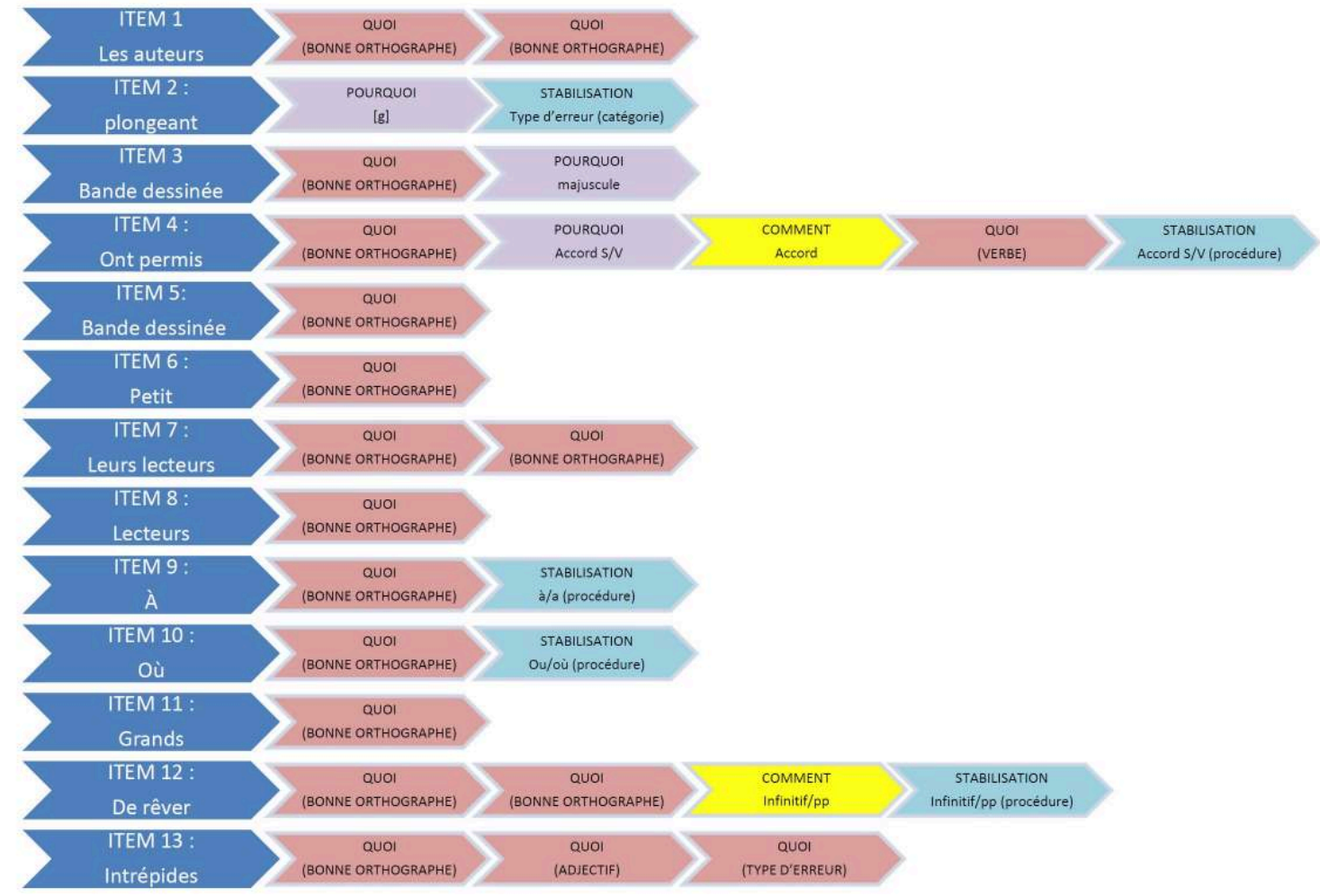

Figure 6 : Exemple de structuration d'une séance de correction de dictée

On pourrait interpréter ce résultat de la manière suivante : l'orthographe est considérée comme un ensemble de savoirs ponctuels à rappeler; ce qui est demandé aux élèves est d'étiqueter plutôt que de conceptualiser (métatermes étiquettes $v s$. métatermes concepts), de répondre aux questions de l'enseignant plutôt que de se questionner et raisonner sur le fonctionnement de la langue. Les pratiques mobilisées relèvent bien d'une littéracie mais pas de celle qui est attendue dans ces situations scolaires d'enseignement-apprentissage de l'orthographe ainsi que nous l'avons déjà analysé auprès d'un public d'étudiants en master MEEF (Lavieu-Gwozdz \& Pagnier 2017)

\section{Des moments discursifs qui visent la procédure /COMMENT/}

Là encore, comme pour la catégorie précédente, la forme linguistique ne présage pas de ce qui est attendu et la forme en comment n'interroge donc pas toujours sur le comment puisque l'enseignant autorise des interventions d'élèves de différents ordres ou niveaux, ainsi que l'illustre ce premier extrait :

\section{Extrait $n^{\circ} 3$}

M : $\quad$ avec le sujet ! c'est parti // Mathis nous trouve le sujet comment on trouve le sujet dans une phrase? 


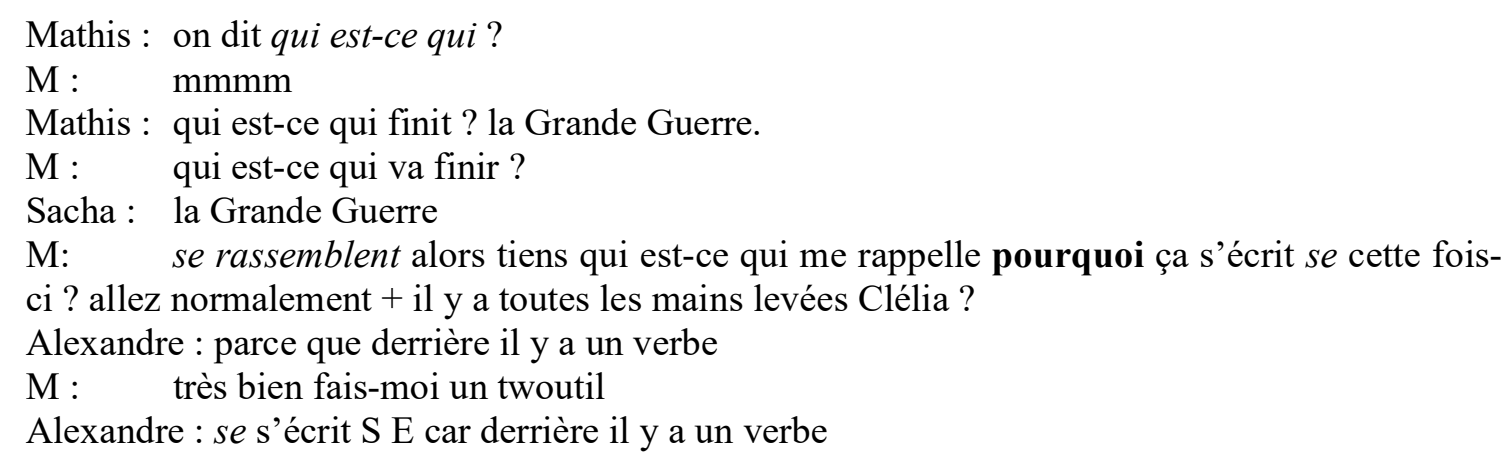

Si nous nous arrêtons sur ce premier extrait pour illustrer notre catégorie en /COMMENT/, nous remarquons (en gras dans l'extrait) que l'enseignante formule aussi bien une question en /comment/ (Comment on le trouve le sujet dans une phrase ?) qu'une question en /pourquoi/ (Pourquoi ça s'écrit $S E$ ). On pourrait alors supposer que les attentes ne sont pas les mêmes. Or, si l'on regarde les réponses des deux élèves Mathis et Alexandre, toutes deux conviennent à l'enseignante (ou du moins aucun écart n'est signalé). Ainsi, bien que la forme de la question soit différente linguistiquement la réponse des élèves vise dans les deux cas la procédure. Dans le premier cas, Mathis énonce, récite une procédure (la question sémantique en qui est-ce qui ?) pour identifier le sujet et dans le second cas, Alexandre mobilise une procédure de repérage, de localisation : c'est parce qu'il y a un verbe derrière «se » qu'on l'écrit SE mais le terme de « verbe pronominal » n'apparait pas et la catégorie n'est pas construite.

\section{Extrait $n^{\circ} 4$}

$\mathrm{M}: \quad$ on termine + ont permis + alors au début vous êtes deux à avoir mis $O N$ alors pourquoi finalement vous avez changé +

Lucas : parce que c'est avoir

M : $\quad$ d'accord + pourquoi vous mettez un $s$ à permis +

Lucas : parce qu'on peut dire permise

La première question formulée en pourquoi ne relève pas de notre catégorie visant le fonctionnement de la langue /POURQUOI/ dans la mesure où c'est davantage l'identification qui fait réponse (validée), en l'occurrence la reconnaissance d'une des formes du verbe avoir. En effet, à la question «pourquoi il fallait mettre ONT et non ON ? », l'enseignante accepte la réponse en "parce que c'est avoir» sans demander aux élèves d'expliciter ce que signifie " parce que c'est avoir », c'est-à-dire pourquoi cette identification est nécessaire, ni comment elle a été réalisée. Il est fort probable que cette réponse témoigne d'une compréhension du raisonnement grammatical à conduire qu'il n'est peut-être pas nécessaire de développer plus avant pour certains élèves (souvent ceux qui participent) mais les conditions auxquelles ce discours peut être pleinement saisis par l'ensemble des élèves et notamment les plus en difficulté méritent d'être interrogées. Le questionnement conduit sur l'item semble pourtant indiquer que la distinction n'est pas acquise par tous et donc l'intervention didactique nécessaire. Le questionnement vise ainsi très fréquemment la vérification de l'application de procédures et de la mobilisation de savoirs supposés maitrisés par tous les élèves. Ainsi, à la seconde question également introduite par «pourquoi » dans l'extrait précédent, la réponse de l'élève pour justifier de la présence du $S$ à permis est également opaque ("parce qu'on peut dire permise ») et n'est pas de nouveau "dépliée » pour que la procédure énoncée le soit explicitement en tant que telle. Ainsi, les procédures appliquées ne donnent jamais lieu à un réel test syntaxique envisageant deux hypothèses pour en valider une et invalidée l'autre. Dans les deux cas de figure, on reste dans l'implicite et aucun des exemples n'est reconfiguré dans le système orthographique du français par exemple pour distinguer le fonctionnement du verbe 
permettre et celui du participe ou le fonctionnement du pronom réfléchi et celui du déterminant démonstratif ou encore celui d'une préposition et celui d'un verbe. En somme, la nature de l'activité langagière et cognitive qu'implique le questionnement grammatical est rendue peu visible dans les interactions, du moins pour celui qui n'est pas en mesure de rétablir l'ensemble de l'implicite sur lequel elles fonctionnent.

Les analyses doivent encore être conduites sur un corpus plus conséquent avant de pouvoir identifier d'éventuels liens entre des proportions significativement différentes et le profil de la classe et/ou d'enseignants. Enfin, les formes linguistiques de ce questionnement sont marquées par une indistinction des introducteurs de questionnement (indifféremment «pourquoi » ou « comment ») d'une part, et par une formulation synthétique des réponses marquée par un fort implicite discursif qui témoigne certainement de ce qui parait évident, mais dont on peut douter qu'il le soit pour tous.

\section{Des moments discursifs qui visent le fonctionnement de la langue : /POURQUOI/}

Dans cette catégorie de discours, nous avons regroupé des extraits d'interactions qui visent des questionnements de type «pourquoi » c'est-à-dire que l'enseignante demande de justifier, d'argumenter les réponses en recourant au fonctionnement du système qu'est la langue, d'en convoquer son mode de fonctionnement. Ainsi, nous avons sélectionné ici des questions du type «Pourquoi ce mot prend un $s$ et pas -ent ?». L'enseignante invite les élèves à mobiliser leur connaissance sur la langue, par exemple sur l'opposition de catégorie grammaticale telle que l'opposition nom/verbe ou encore sur l'opposition nom propre/nom commun comme l'illustre l'extrait suivant :

\section{Extrait $n^{\circ} 5$ :}

M: $\quad$ alors + la grande Guerre nous + quand on a réfléchi au texte, on l'a écrit avec des majuscules + alors pourquoi on l'a écrit avec des majuscules + Max ?

Léa : parce que c'est la première guerre mondiale

M : $\quad$ c'est le nom qu'on a donné à la première guerre mondiale alors ça vous ne pouviez pas le savoir parce qu'en histoire vous n'êtes pas encore là mais on l'a appelée souvent on dit tiens c'est la Grande Guerre alors la Grande Guerre malheureusement c'est parce que c'est la guerre où il y a eu le plus de morts alors on l'appelle la Grande Guerre et si on l'a appelée comme ça + ça devient du coup un nom...

Léa: propre

M : $\quad$ ça devient un nom propre.

Dans ce cas, l'enseignant prend en charge ce discours de stabilisation des savoirs qu'elle fait compléter par une élève.

\section{Conclusion}

Nous avons cherché à identifier les activités discursives auxquelles donnait lieu le questionnement - autour des activités linguistiques - conduit en classe. L'analyse des premières données traitées ne montre pas de différences significatives selon qu'il s'agisse de classes en REP ou non REP ou de classes utilisant un dispositif innovant comme la Twictée ou non. Évidemment, ces premières conclusions demanderont à être confirmées, notamment par l'analyse de davantage de séances. Nous pouvons en revanche identifier des tendances communes. 
Ainsi, d'après les manuels analysés, d'après les échanges langagiers recueillis dans la salle de classe, nous pouvons craindre que tous les élèves ne perçoivent pas la finalité des exercices d'orthographe ni des corrections de dictées, ce qui explique certainement au moins pour partie le recours à des procédures inadaptées et à une manière "ordinaire " de catégoriser qui ne relève pas de la catégorisation scientifique (du moins scolaire). Les premiers résultats montrent ainsi que pour une part importante des questionnements, l'activité orthographique demeure une activité tendue par une finalité que l'on qualifierait volontiers d'autotélique (Belin 2002; Delarue-Breton 2012) tant elle s'épuise dans la réalisation même de l'activité : faire de l'orthographe, c'est étiqueter. Ainsi, le questionnement tel qu'il a été observé en classe donne le plus souvent lieu à de l'identification ou du repérage (de catégories, de formes correctes, de mots ou groupes de mots...) sans que les procédures et les finalités de ces identifications ne soient explicitées. De fait, ce sont les possibilités d'appropriation du questionnement qui se posent pour les élèves les plus en difficulté confrontés à un discours dont ils doivent rétablir les implicites pour y participer et se l'approprier. Par ailleurs, les procédures telles qu'elles sont explicitées dans les textes de savoirs (et donc à mobiliser dans les exercices) et dans les interactions en classe sont principalement marquées par le recours systématique aux questions d'ordre sémantique (en qui est-ce qui ? par exemple pour trouver le sujet ou en quoi ? pour identifier le COD), qui est effectivement une procédure enseignée dans l'élémentaire mais le plus souvent, et nous l'avons vu dans les programmes, parmi d'autres procédures. Or, dans les exercices de manuels et dans les interactions, elle fonctionne isolément faisant ainsi reposer la catégorisation sur un jugement de typicalité censément suffisant. Les élèves n'envisagent d'ailleurs que rarement des prolongements. Leur statut de procédure, c'est-à-dire d'outil permettant de mener un «réel» test syntaxique pour répondre à un questionnement sur le fonctionnement de la langue n'est pas mis en discours. Le fondement de ces procédures ne fait pas non plus l'objet d'explicitation (par exemple, pourquoi mettre au féminin un participe passé). Les procédures sont ainsi primarisées en ce sens qu'elles ne sont pas ressaisies, retravaillées, secondarisées ${ }^{7}$ dans une perspective d'apprentissage de la langue et de son fonctionnement, mais sont extraites et mises en fonctionnement dans un discours premier, comme on pourrait le faire dans un échange ordinaire, conversationnel.

Un des résultats majeurs de notre analyse, et qui ouvre des perspectives de recherches et de formations fécondes, tient aux formes linguistiques du questionnement qui ne sont pas contraignantes ou du moins ne guident pas explicitement l'activité discursive. En effet, si les programmes étayent peu le questionnement, les manuels et les interactions en classe ne le spécifient, ne le guident, ni ne l'articulent davantage. Il semble qu'une des clefs de l'efficience est donc certainement à chercher dans le questionnement et le statut d'objet d'analyse scientifique et d'objet de formation qu'il faut, lui, construire. Dans cette perspective, nous poursuivrons l'analyse de l'articulation entre les discours instructeurs (Bernstein 2007) d'élaboration et de stabilisation (Lavieu-Gwozdz \& Pagnier 2020) dans le polylogue scolaire mais aussi l'appropriation individuelle de ces interactions collectives à partir de l'examen d'entretiens méta-orthographiques conduits avec les élèves.

\footnotetext{
${ }^{7}$ La notion de « secondarisation » (voir Bautier \& Goigoux 2004) implique simultanément décontextualisation et adoption d'une autre finalité. Or, les procédures comme les catégories sont ici «primarisées ». L'appariement se réalise sur la base de la vérification dans une liste mentale d'éléments et/ou sur le sens que les élèves donnent aux éléments stéréotypiques de cette catégorie (la catégorie «nom» regroupe les éléments désignant des choses, la catégorie «sujet» regroupe des éléments qui désignent celui qui fait l'action, la catégorie «verbe » indique l'action, et ainsi de suite). Cette procédure d'étiquetage relève bien d'un usage littéracié de la langue. Pour autant, elle s'écarte de l'activité d'analyse que vise l'enseignement orthographique, notamment aujourd'hui.
} 


\section{Références}

AUDION Lionel, 2015, Acceptabilité et opérations métalinguistiques chez les élèves de cycle 3 , Thèse de doctorat, Université de Nantes.

AUDION Lionel, 2017, «Encourager la posture métalingusitique des élèves de cycle 3 : pourquoi ? Comment ? », Le français aujourd'hui $\mathrm{n}^{\circ} 198$, p. 39-51.

BAUTIER Élisabeth, 1997, « Les pratiques socio-langagières dans la classe de français ? Quels enjeux ? Quelles démarches ?», Repères, $\mathrm{n}^{\circ} 15$.

BAUTIER Élisabeth, 2001, "Pratiques langagières et scolarisation», Revue française de pédagogie, $\mathrm{n}^{\circ} 137$, p. 117-161.

BAUTIER Élisabeth, 2005, «Formes et activités scolaires, secondarisation, reconfiguration, différenciation sociale ", dans Nicole Ramognino et Pierrette Vergès (dir.), Le français hier et aujourd'hui : Politiques de la langue et apprentissages scolaires. Hommage à Viviane Isambert-Jamati, Aix-en-Provence : Publications de l'Université de Provence, p. 49-68.

BAUTIER Élisabeth, 2008, Ce que l'école fait aux individus. Colloque de Sociologie de l'éducation dans le cadre de la journée scientifique de l'Université de Nantes: «Pratiques de travail et pratiques langagières scolaires : constructions au quotidien des inégalités sociales d'apprentissage ».

BAUTIER Élisabeth, 2009, «Quand le discours pédagogique entrave la construction des usages littéraciés du langage », Pratiques : Écrits et savoirs, n $143-144$, p.11-26.

BAUTIER Élisabeth \& ROCHEX Jean-Yves, 2004, "Activité conjointe ne signifie pas signification partagée », Raisons éducatives, ${ }^{\circ}$ 8, p. 199-220.

BAUTIER Élisabeth et RAYOU Patrick, 2009, Les inégalités d'apprentissage : programmes, pratiques et malentendus scolaires, Paris, France : Presses universitaires de France.

BELIN Emmanuel, 2002, Une sociologie des espaces potentiels. Logique dispositive et expérience ordinaire, Bruxelles : De Boeck Université.

BERNSTEIN Basil, 2007, Pédagogie, contrôle symbolique et identité : théorie, recherche, critique. Presses Université Laval.

BOUTET Josiane, 2005, « Pour une activité réflexive sur la langue », Le français aujourd'hui, $\mathrm{n}^{\circ} 148(1)$, p. 65-74.

BRISSAUD Catherine \& MORTAMET Clara, 2015, La dictée, une pratique sociale emblématique, Glottopol ${ }^{\circ} 26$, Université de Rouen.

CÈBE Sylvie \& GOIGOUX Roland, 2009, Lector \& lectrix, apprendre à comprendre les textes narratifs, Retz.

CHARTRAND Suzanne-Geneviève (dir.), 2016, Mieux enseigner la grammaire. Pistes didactiques et activités pour la classe, Montréal : Pearson, coll. «ERPI- Éducation ».

CHERVEL André, 1988, Pour une histoire des disciplines scolaires. Histoire de l'éducation, $\mathrm{n}^{\circ} 38$, p. 59-119.

COGIS Danièle, 2003, "L'orthographe, un enseignement en mutation », Le français aujourd'hui, $\mathrm{n}^{\circ}$ 141(2), p.118-122.

DELARUE-BRETON Catherine, 2012, « Dispositifs et logiques dispositives : perception des enjeux et inégalités scolaires », in Marie-Laure Elalouf, Aline Robert, Anissa Belhadjin \& Marie-France Bishop (dir.), Les didactiques en question: état des lieux et perspectives pour la recherche et la formation, Bruxelles : De Boeck, p. 120-130.

DELARUE-BRETON Catherine, 2014, «Dialogue scolaire, dialogisme et évènements d'énonciation : malentendus et inentendus au sein de la classe », Études de Linguistique Appliquée, $\mathrm{n}^{\circ}$ 173, p. 87-97.

DELARUE-BRETON Catherine, 2015, « D'un document à l'autre : circulation des savoirs et déambulations différenciées des élèves ", Didactiques en pratique, ${ }^{\circ}$ 1, p. 18-24. 
DELARUE-BRETON Catherine, 2017, «Activité dialogique à l'école : observations sur l'élaboration des significations au sein du dialogue scolaire », Recherches en didactiques des langues et des cultures, $\mathrm{n}^{\circ} 14-2$. http://rdlc.revues.org/1875

DENIZOT Nathalie \& BISHOP Marie-France, 2016, «Explorer les manuels scolaires », Le Français Aujourd'hui n 194, p. 5-11.

DOUAIRE Jacques, ELALOUF Marie-Laure \& POMMIER Patrick, 2005, «Savoirs professionnels et spécificités disciplinaires analyse de mises en commun dans trois disciplines », Grand N, n 75, p. 45-57.

DUEZ Guillaume \& RISSELIN Karine, 2017, «L'enseignement de la langue au cycle 4 : des programmes entre rupture curriculaire et continuité didactique », Le Français Aujourd'hui, $\mathrm{n}^{\circ} 198$, p. 25-35.

ELALOUF Marie-Laure, 2005, « De la $6^{\mathrm{e}}$ à la $1^{\text {re }}$, comment mobilisent-ils leurs connaissances sur la langue dans des tâches d'explication? », Pratiques, $\mathrm{n}^{\circ}$ 125-126, p. 157-178.

GAGNON Roxane \& PERRET Claudie, 2009, «Pratiques d'observation de la langue en France : quelles évolutions ? Quels obstacles? », dans Joachim Dolz \& Claude Simard (dir.), Pratiques d'enseignement grammatical, Québec : Presses universitaires Laval, p. 49-72.

GARCIA-DEBANC Claudine, PAOLACCI Véronique \& BOIVIN Marie-Claude, 2014, «L'étude de la langue : des curricula aux pratiques observées », Repères n ${ }^{\circ} 49$, p. 7-32.

GAUVIN Isabelle, 2014, Le rapport à la grammaire et à la didactique de la grammaire de futurs enseignants de français langue première et secondaire: un aperçu, http://www.shsconferences.org/articles/shsconf/pdf/2014/05/shsconf cmlf14 01343.pdf.

GIORDAN André et DE VECCHI Gérard, 1987, Les origines du savoir. Des conceptions des apprenants aux concepts scientifiques., Lausanne \& Paris, Delachaux \& Niestlé.

HUBERT Bruno, 2016, "Formation des enseignants : le casse-tête de la grammaire », Le français aujourd'hui, $\mathrm{n}^{\circ} 192$, p. 117-129.

JORRO Anne et MERCIER-BRUNEL Yan, 2011, « Les gestes évaluatifs de l'enseignant dans une tâche de correction collective », Mesure et évaluation en éducation, 34(3), p. 2750 .

LAVIEU-GWOZDZ Belinda \& PAGNIER Thierry, 2017, «Quelles pratiques littéraciées sont mobilisées par les futurs PE », Lidil n ${ }^{\circ} 56$, https://journals.openedition.org/lidil/4806.

LAVIEU-GWOZDZ Belinda \& PAGNIER Thierry, 2020, «La mise en discours du savoir orthographique : que se passe-t-il dans la salle de classe ? », Actes du Congrès Mondial de Linguistique Française 2020, DOI : 10.1051/shsconf/20207807011.

MAULINI Olivier, 2004, «Un enjeu invisible de l'innovation pédagogique : l'institution du questionnement », dans : Jean-Paul Bronckart (éd.), Transformer l'école, Louvain-laNeuve, Belgique : De Boeck Supérieur, p. 127-146.

NADEAU Marie et FISHER Carole, 2011, «Les connaissances implicites et explicites en grammaire: quelle importance pour l'enseignement? Quelles conséquences?», Bellaterra Journal of Teaching \& Learning Language \& Literature, $n^{\circ} 4(4)$, p. 1-31.

PELLAT Jean-Christophe, 2017, Quelle grammaire enseigner? Paris : Hatier ( $3{ }^{\text {ème }}$ édition).

RAMOGNINO Nicole, 2007, "Normes sociales, normativités individuelle et collective, normativité de l'action ", Langage et société, $\mathrm{n}^{\circ} 119$, p. 13-41.

ROCHEX Jean-Yves \& CRINON Jacques (dir.), 2011, La construction des inégalités scolaires. Au cour des pratiques et des dispositifs d'enseignement, Rennes : PUR

ULMA Dominique, 2016, "Construction de savoirs grammaticaux et conceptualisation », Le français aujourd'hui, $\mathrm{n}^{\circ}$ 192, p. 97-106. 


\section{Programmes \& manuels}

Ministère de l'Éducation nationale, 2015, Bulletin Officiel de l'Éducation Nationale, Spéciale $\mathrm{n}^{\circ} 11,26$ novembre 2015, https://cache.media.eduscol.education.fr/file/ programmes 2018/20/2/Cycle 3 programme consolide 1038202.pdf

Terre des mots, CMI (cycle 3), Nathan, 2016, sous la direction de A. Bentolila.

Explorons la langue, CM1 (cycle 3), Belin, 2017, sous la direction de Gérard Jeangrand \& Nathalie Dion-Samy.

Interlignes, CM1 (cycle3), Sed, 2017, sous la direction de Catherine Castera, Pascale BrailletPasquereau \& Isabelle Verkindre-Ripard. 


\section{GLOTTOPOL}

Revue de sociolinguistique en ligne

Comité de rédaction : Michaël Abecassis (University of Oxford), Salih Akin (Université de Rouen Normandie), Sophie Babault (Université de Lille), Aude Bretegnier (Université du Mans), Claude Caitucoli, Véronique Castellotti (Université de Tours), Régine DelamotteLegrand (Université de Rouen Normandie), Alexandre Duchêne (Université de Fribourg), Valentin Feussi (Université d'Angers), Robert Fournier (Carleton University, Ottawa), Stéphanie Galligani (Université Grenoble Alpes), Médéric Gasquet-Cyrus (Université AixMarseille), Emmanuelle Huver (Université de Tours), Normand Labrie (Université de Toronto), Foued Laroussi (Université de Rouen Normandie), Benoit Leblanc (Université du Québec à Trois-Rivières), Mylène Lebon-Eyquem (Université de la Réunion), Fabienne Leconte (Université de Rouen Normandie), Gudrun Ledegen (Université de Rennes), Danièle Moore (Simon Fraser University, Vancouver), Clara Mortamet (Université Jean Monnet, Saint Etienne), Alioune Ndao (Université Cheik Anta Diop, Dakar), Isabelle Pierozak (Université de Tours), Cécile Van den Avenne (Université Sorbonne Nouvelle, Paris 3).

Rédactrice en chef : Clara Mortamet.

Directrice de publication : Fabienne Leconte.

Comité scientifique: Michelle Auzanneau (Université de Paris), Margaret Bento (Université de Paris), Jacqueline Billiez (Université de Grenobles Alpes), Philippe Blanchet (Université de Rennes), Jean-Michel Eloy (Université d'Amiens), Françoise Gadet (Université Paris Nanterre), Monica Heller (Université de Moncton), Caroline Juillard (Université de Paris), Jean-Marie Klinkenberg (Université de Liège), Marinette Matthey (Université Grenoble Alpes), Marie-Louise Moreau (Université de Mons-Hainault), Robert Nicolaï (Université Côte d'Azur), Didier de Robillard (Université de Tours), Valérie Spaëth (Université Sorbonne nouvelle), Claude Truchot (Université de Strasbourg), Daniel Véronique (Université AixMarseille).

\section{Comité de lecture pour ce numéro :}

Nathalie Auger, Michèle Auzanneau, Margaret Bento, Stéphane Bonnery, Josiane Boutet, Lucile Cadet, Danièle Cogis, Claudine Garcia-Debanc, Marc Debono Régine Delamotte, JeanFrançois De Pietro, Marie-Laure Elalouf, Valentin Feussi, Laurent Gajo, Emmanuelle Huver, Christian Lagarde, Mylène Lebon-Eyquem, Fabienne Leconte, Gudrun Ledegen, Nadia Maillard, Maira Mamede, Bruno Maurer, Clara Mortamet, Fanny Rink, Valerie Spaëth, Marielle Rispail, Françoise Ropé, Eguzki Urteaga, Cécile Van den Avenne, Daniel Véronique 